Ann. Sci. forest., 1981, 38 (2), 237-258

\title{
Modifications des caractéristiques physico-chimiques d'un sol brun acide des Ardennes primaires par la monoculture d'Epicéa commun
}

\author{
C. NYS \\ avec la collaboration de Dominique Vairelees, Jitka Lhomme et D. Gelhaye \\ Station de Recherches sur les Sols forestiers et la Fertilisation \\ Centre de Recherches forestières de Nancy, I.N.R.A., \\ Champenoux, 54280 Seichamps
}

\begin{abstract}
Résumé
Cet article résume les modifications des caractéristiques physico-chimiques d'un sol brun acide, sous un peuplement forestier feuillu, développé sur des limons reposant sur les schistes du Revinien dans les Ardennes primaires.

L'introduction de l'Epicéa (Picea abies) a provoqué un début de podzolisation. L'humus de type mull acide sous les feuillus est moder-mull sous les Epicéas. L'acidité libre et l'indice de redistribution de l'alumine et du fer libres sont plus élevés sous les Epicéas.

Les réserves des éléments échangeables, mobilisables et les immobilisations dans la biomasse aérienne sont semblables dans les deux écosystèmes. A court terme il n'y a pas diminution de la fertilité de la station par enrésinement.

Le bilan isoquartz a été appliqué aux éléments totaux. Il n'y a pas de modification importante des réserves minérales. Lessivage et altération seraient plus intenses sous les résineux mais l'accumulation d'argiles et l'altération possible de l'horizon $\mathrm{C}$ (horizon de référence pour le calcul isoquartz) sous les Epicéas pose le problème de limite et d'exactitude d'un tel calcul.
\end{abstract}

Depuis plus de 30 ans la politique forestière nationale encourage les plantations d'essences résineuses dont l'économie a un réel besoin (pâte à papier et bois de trituration). Mais la monoculture de conifères provoque sur le sol des modifications physico-chimiques et biologiques.

De nombreuses études ont été effectuées sous des peuplemens résineux artificiels (Genssler, 1959 ; Holmsgaard et al., 1961 ; Duchaufour \& Bonneau, 1961 ; SChlenker et al., 1969 ; Miehlich, 1970 ; Nihlgard, 1972 ; Brethes \& NYS, 1975 ; Nys, 1976 ; Bonneau et al., 1976 ; Compte rendu D.G.R.S.T., 1977 ; RICHERT, 1978 ; Paternoster, 1979 ; Guillaud, 1979) afin de savoir sill y a baisse de fertilité des sols qui les portent, et s'il existe ou non des risques d'altérations irréversibles et de « dégradation 》 au sens pédogénétique. Des mises au point sur les conséquences pédologiques des monocultures de résineux ont été publiées (MaNiL, 1966 ; BonNEAU, 1973 ; NoIRFALISE \& VANESSE, 1975 ; BonNeau, 1978).

Ces travaux ne permettaient pas de répondre complètement à la question posée. C'est pourquoi nous menons une étude comparée dans différents écosystèmes forestiers, en particulier dans les Ardennes Françaises où nous essayons de réaliser une étude 
globale du fonctionnement d'une futaie d'Epicéa et d'un peuplement feuillu représentant la forêt originelle de cette région. sols.

Cet article rend compte des résultats de l'étude comparative de l'évolution des

\section{I. - Caractérisation des deux stations étudiées}

La station est située en forêt domaniale de Château-Regnault dans les Ardennes primaires. L'altitude est de $390 \mathrm{~m}$ et la pente faible (4 à 6 p. 100). Le climat de type montagnard est froid et humide. Les précipitations moyennes annuelles sont de $1100 \mathrm{~mm}$. La température d'environ 8 "C.

\section{I.11. - Les peuplements}

\section{I.1. - Ecologie des peuplements}

Le peuplement feuillu est un taillis-sous-futaie. Les réserves (76 tiges/ha) sont constituées par 90 p. 100 de Chêne (Quercus sessiliflora Salisb.) et 10 p. 100 de Hêtre (Fagus sylvatica L.). Le taillis (6 150 tiges/ha) est âgé de 28 ans et pauvre. Deux essences dominantes le composent : le Sorbier des oiseleurs (Sorbus aucuparia L.) et le Bouleau (Betula verrucosa E.). Les essences d'accompagnement sont le Chêne, le Coudrier (Corylus avellana L.) et la Bourdaine (Rhamnus frangula L.), la Fougère aigle (Pteris aquilinum) et la Ronce (Rubus sp.) sont les espèces de la strate herbacée.

Le peuplement d'Epicéa [Picea excelsa (Lam.) Link] était âgé de 45 ans en 1976. Sa densité est de 1700 tiges par hectare. Le peuplement n'a jamais été éclairci.

\section{I.12. - Les profils du sol}

Nous avons effectué dans chaque station, après la cartographie des sols, les descriptions de 5 profils du sol. Ceci nous permet de dégager la parenté de ces deux stations feuillues et résineuses et de préciser les modifications morphologiques dues à la monoculture d'Epicéa. Nous considérons qu'avant l'introduction des épicéas il y a 45 ans, les deux stations étaient analogues.

\section{I.121. - Le profil sous les feuillus ou sol de référence}

La litière des feuilles de l'année est peu épaisse (moins de $1 \mathrm{~cm}$ ) et discontinue. Elle recouvre un horizon Ao des feuilles en voie de décomposition très peu épais et local.

0-5 cm : dans l'horizon A11, la structure est grumeleuse friable, la texture limoneuse. Un feutrage de fines racines de graminées envahit cet horizon.

$5-8 \mathrm{~cm}$ : A12 incorporation de matière organique plus ou moins régulière.

L'humus est de type mull acide.

8-25 $\mathrm{cm}$ : horizon de transition avec l'horizon (B) de structure polyédrique de petites dimensions et peu stable. Texture limoneuse avec quelques gravillons plats de schiste et quelques cailloux gréseux.

$25-50 \mathrm{~cm}$ : horizon $(B)$ de couleur brun. La structure est à tendance polyédrique peu stable. La texture limoneuse.

La teneur en gravillons schisteux augmente avec la profondeur. 

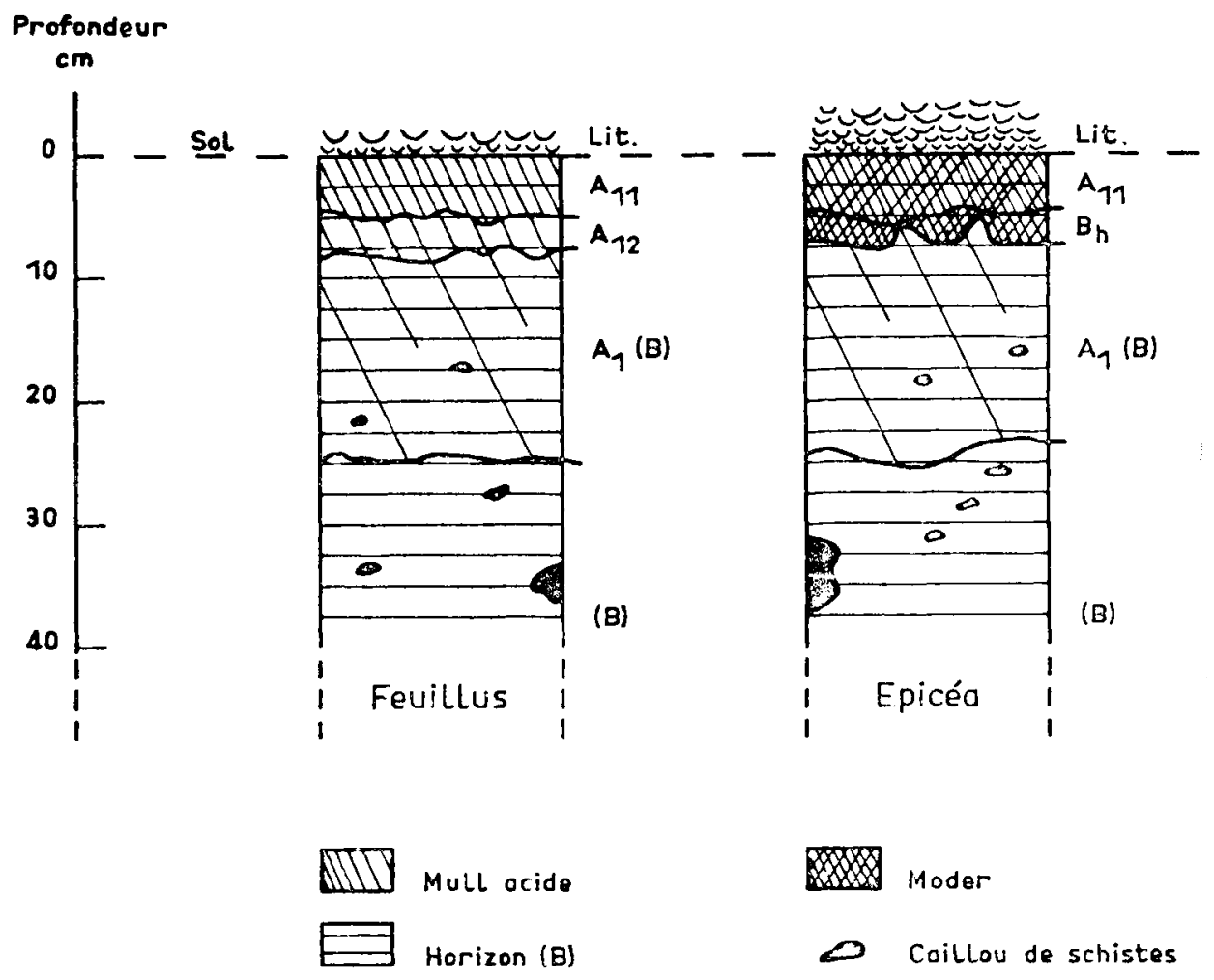

Mull ocide

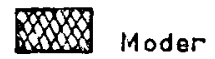

Horizon (B)

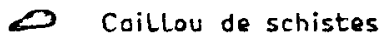

FIGURE 1

Schémas des profils du sol Soils profile diagram

$50-85 \mathrm{~cm}$ : transition vers l'horizon $\mathrm{C}$, couleur passe du brun au gris bleu.

$85-100 \mathrm{~cm}$ : horizon $\mathrm{C}$ : zone gris-bleu, altération des schistes avec quelques tâches rouilles.

$100 \mathrm{~cm}$ : schistes bleus du Révinien en place.

Le sol est de type brun acide.

\subsection{2. - Le sol sous les Epicéas}

La morphologie du profil type sous les épicéas se différencie du précédent uniquement par ses horizons organiques (25 premiers centimètres).

La litière composée d'aiguilles d'Epicéa est plus épaisse et continue, de même pour le Ao.

$0-5 \mathrm{~cm}:$ l'horizon $\mathrm{A} 1$, plus sombre que le précédent, a une structure à tendance particulaire. 
$5-7 \mathrm{~cm}$ : un horizon $\mathrm{Bh}$, infiltration de matière organique, de couleur marron chocolat, s'est développé, il est ondulé et d'épaisseur variable.

$7-25 \mathrm{~cm}$ : horizon de transition plus riche en matière organique que son homologue sous feuillus.

L'humus est de type moder-mull et le sol de type brun ocreux.

La monoculture d'Epicéa a provoqué des modifications morphologiques des humus. Quelles sont donc les modifications des principales caractéristiques physiques et chimiques?

\section{I.2. - Les caractéristiques physico-chimiques des profils types}

\section{I.21. - Méthodes analytiques}

Dans chacune de nos deux stations nous avons prélevé 5 profils du sol divisés en 10 horizons. Pour chaque échantillon ont été déterminés : les taux d'humidité $(105$ "C), les densités apparentes (mesurées : d'm).

Pour chaque station 3 profils ont été totalement analysés. Les résultats donnés dans cette publication correspondent aux moyennes des 3 profils pour les feuillus et de 2 pour les épicéas (un profil ne s’avérant pas parfaitement homogène verticalement).

Les principales méthodes d'analyse sont décrites dans Duchaufour (1970). Ce sont principalement les dosages de : Carbone (C) par la méthode Anne, l'azote total (Kjeldahl), $\rho \mathrm{H}$ eau, acidités libres (eau), acidités d'échange ( $\mathrm{KCl}$ normal) et acidités totales (résine $\mathrm{H}^{+}$) par titration à la soude; les éléments échangeables (extraction acétate d'ammonium), la capacité totale d'échange (méthode Metson), les éléments mobilisables (E.D.T.A. + tétraphénylborate (VEDY, 1973)), les éléments libres fer et aluminium (Tamm + hydrosulfite de sodium), la silice amorphe $(\mathrm{NaOH})$ et les éléments totaux (déterminés par fusion alcaline au métaborate de strontium) sur les fractions grossières (supérieure à 2 micromètres) et les argiles granulométriques.

Nous avons d'autre part déterminé la composition minérale des échantillons : micas, feldspaths et quartz. Le principe de cette méthode est la dissolution chimique différentielle (JACKSON, 1974) : après séparation des argiles (fraction granulométrique inférieure à 2 micromètres) les micas sont dissous par le pyrosulfate de sodium à chaud, les feldspaths du culot restant sont dissous à froid $\left(15^{\circ} \mathrm{C}\right)$ par l'acide fluorosilicique. Le résidu, après vérification aux R.X., est le quartz déterminé par pesée.

\section{I.22. - Comparaison des caractéristiques pédologiques}

Les principales caractéristiques des sols sont décrites dans le tableau 1 et les figures 2 et 3 .

D'une manière générale l'ensemble de ces analyses de caractérisation confirme l'homogénéité des deux stations et leur bonne parenté et montre qu'il n'y a pas de modification dans les horizons minéraux (plus de $25 \mathrm{~cm}$ de profondeur) excepté pour les éléments libres et amorphes.

Par contre nous constatons dans les horizons organiques des modifications systématiques des principales caractéristiques des profils du sol. 


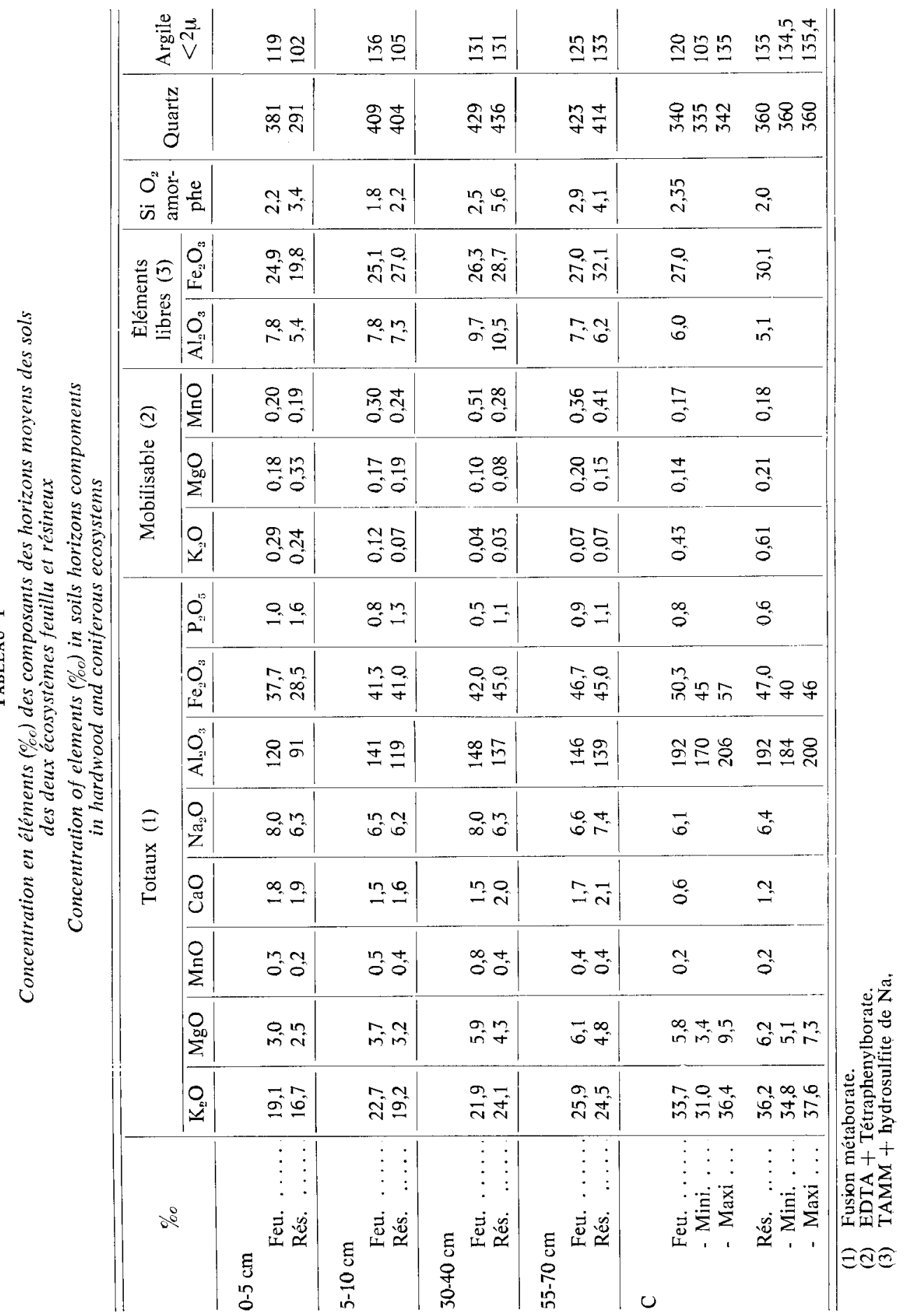



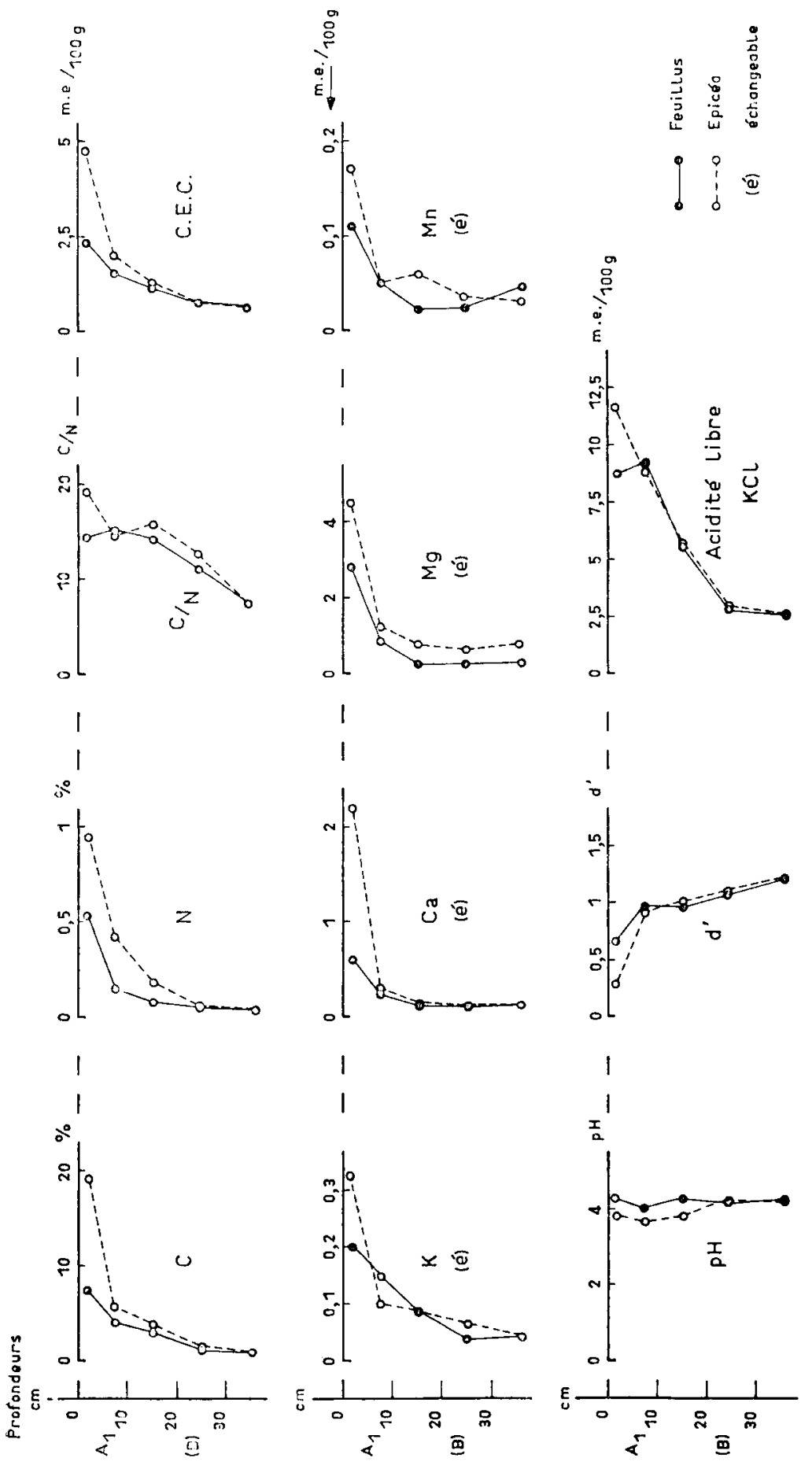
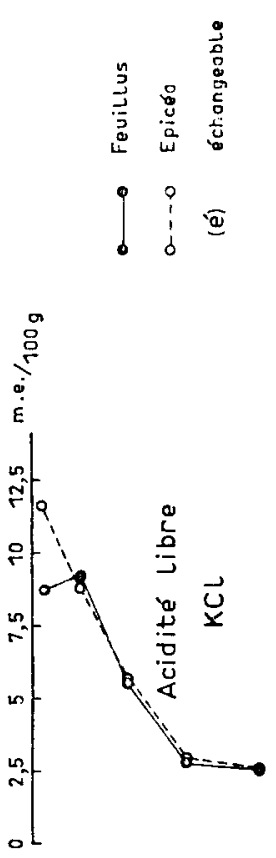

$\sim$
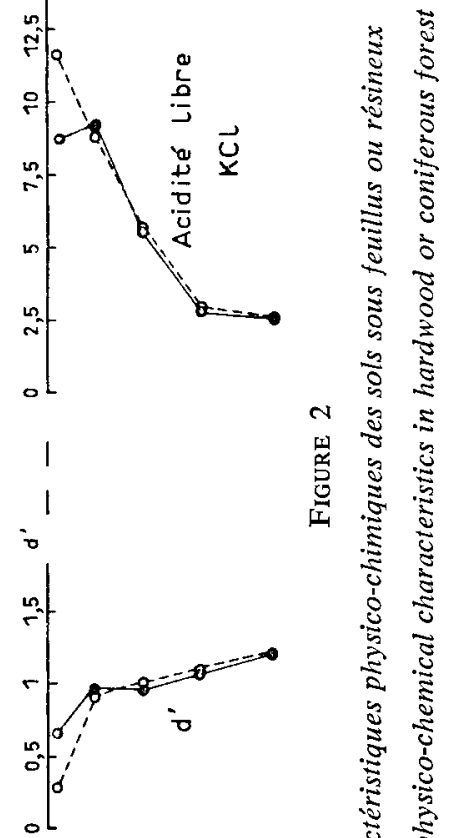

号忽

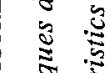

芯

嫣

ป

ह

ฐ

ป

. 
MODIFICATIONS DU SOL PAR LA CULTURE D'ÉPICEA

243
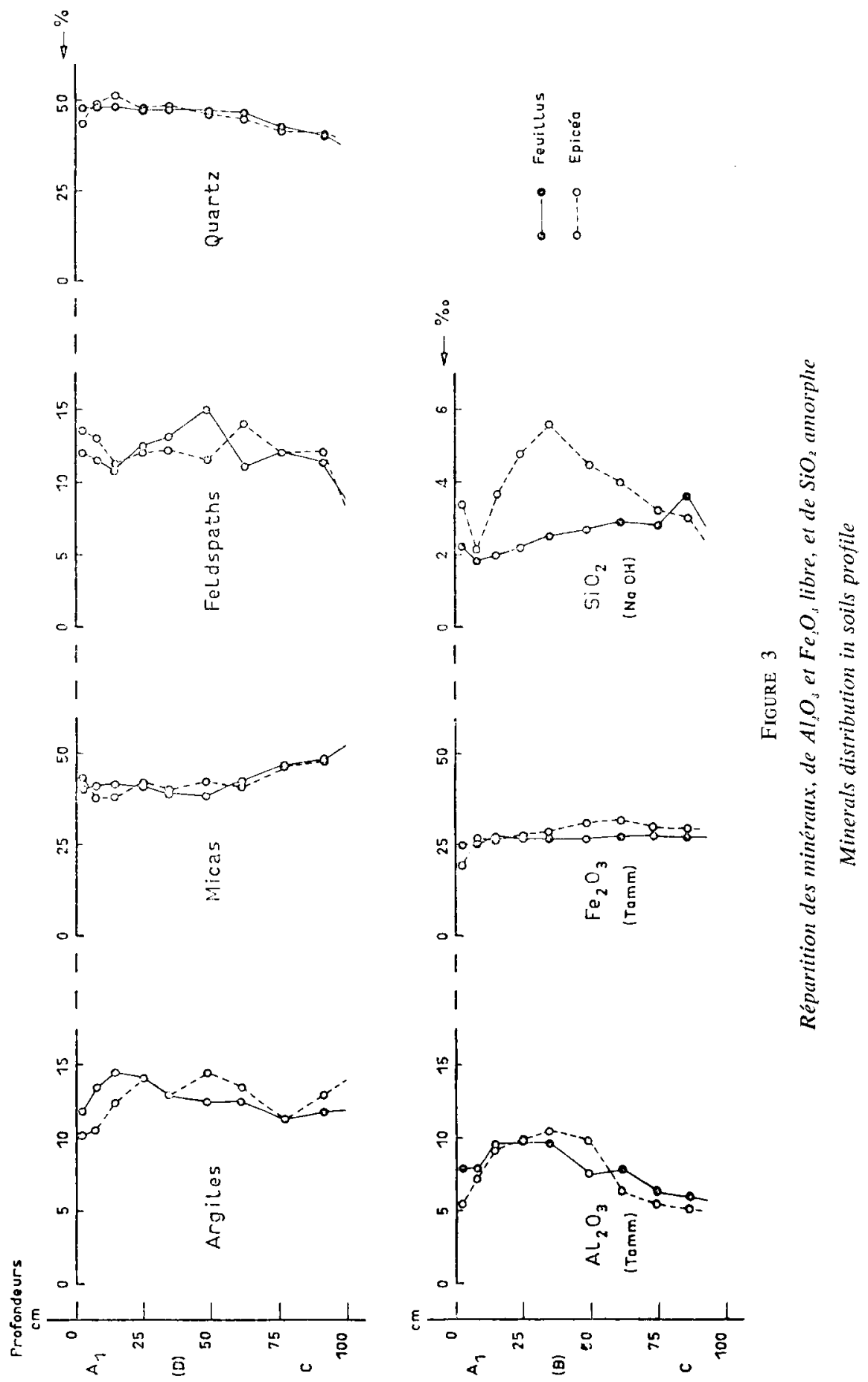


\section{I.221. - Carbone, Azote et $C / N$}

Les deux éléments carbone et azote, apportés aux sols par le jeu du cycle biologique (litière, racines) s'accumulent plus sous les épicéas. Les retombées annuelles de litière sont équivalentes (environ $4000 \mathrm{~kg} / \mathrm{ha} / \mathrm{an}$ ), cependant la moins bonne minéralisation sous les résineux (Guillaud, 1979) a pour conséquence son accumulation dans la litière et l'horizon $\mathrm{A} 1$. Le $\mathrm{C} / \mathrm{N}$ est plus élevé en surface (litière, A1) sous les Epicéas et présente un léger ventre au niveau de l'horizon Bh sous les épicéas.

\section{I.222. - Capacité totale d'échange (C.E.C.)}

Elle est plus élevée dans les horizons de surface sous les épicéas, ceci en liaison avec une teneur plus élevée en matière organique.

\section{I.223. - Eléments échangeables}

Ces éléments du complexe absorbant sont directement disponibles pour l'alimentation minérale des végétaux. Ils sont le reflet du cycle biologique et précisent le niveau de fertilité de la station.

Nous constatons que les teneurs en cations échangeables (calcium, potassium, magnésium et manganèse) sont plus élevées sous les épicéas dans les 25 premiers centimètres de profils (horizons organiques).

\section{I.224. - pH et acidité}

Dans les horizons organiques du sol $\left(\mathrm{L}, \mathrm{A}_{\mathrm{l}}\right)$ le $\mathrm{pH}$ est inférieur d'environ 0,5 unité sous les épicéas. Les différentes formes d'acidité et en particulier celle d'échange décroissent vers la base du profil où elles deviennent égales dans les profils des deux stations. En surface, l'acidité d'échange est plus élevée sous les résineux (RicherT, 1978). Ceci traduit chez l'Epicéa une potentialité plus grande à l'altération des minéraux primaires.

\section{I.225. - Eléments mobilisables et totaux}

Les minéraux primaires s'altèrent sous l'action des eaux de gravité ou liées et de leurs composants organiques, formant le complexe d'altération. Celui-ci est capable de libérer plus ou moins rapidement certains éléments susceptibles alors de recharger le complexe absorbant en éléments échangeables.

Sauf pour le magnésium mobilisable, nous constatons sous les résineux une diminution des concentrations en éléments mobilisables et totaux par rapport aux feuillus.

\section{I.226. - Eléments libres et amorphes}

Nous constatons des teneurs et une redistribution sur l'ensemble du profil différentes entre les feuillus et les épicéas. résineux.

La silice amorphe (extraction $\mathrm{NaOH}$ ) présente des teneurs plus élevées sous les 
Le fer libre et laluminium libre (extraction Tamm + hydrosulfite de sodium) sont redistribués avec des intensités différentes. Les coefficients de redistribution $\mathrm{K}_{\Lambda 1}$ et $\mathbf{K}_{\mathrm{F}:}$ (Souchier, 1971) montrent que ceux-ci sont plus élevés pour les sols sous Epicéa que sous feuillus.

$$
\begin{array}{ll}
\mathrm{K}_{A 1}(\text { Epicéa })=2,0 & \mathrm{~K}_{\mathrm{Al}} \text { (feuillus) }=1,4 \\
\mathrm{~K}_{\mathrm{Fe}}(\text { Epicéa })=1,15 & \mathrm{~K}_{\mathrm{F} .} \text { (feuillus) }=1,0
\end{array}
$$

Remarque : nous constatons que dans l'horizon C la quantité d'éléments libres et amorphes est importante par rapport aux horizons de surface du sol. Ceci confirme que nos stations sont sur une roche mère ancienne, bien différente de celle précédemment étudiée (granite de Millevaches peu altéré) et que l'altération de notre horizon $\mathrm{C}$ de référence est déjà ancienne.

La proportion de fer extractible au réactif de Tamm + hydrosulfite de sodium représente plus de 60 p. 100 du fer total.

\section{I.227. - Les minéraux du sol}

La technique de dissolution sélective des minéraux primaires pour déterminer la teneur en quartz permet en complément d'évaluer les différents constituants minéraux du sol.

La figure 3 montre la similitude minéralogique des deux profils moyens quant à la répartition des teneurs en quartz variant peu autour de $40-50$ p. 100 , des micas 30 à 40 p. 100, des feldspaths 7 à 10 p. 100, des argiles granulométriques 10 à 13 p. 100 .

Les profils se différencient cependant en surface par une diminution sous les épicéas des argiles et micas et une augmentation du quartz et également dans lhorizon $\mathrm{C}$ par une accumulation faible d’argile sous les épicéas qui serait due à un lessivage plus intense.

\section{Conclusion}

Nous constatons que 45 ans de monoculture d'Epicéa a provoqué des modifications qualitatives des principales caractéristiques des sols dans les horizons de surface.

L'acidification, la migration et (ou) l'altération des argiles (inférieures à 2 micromètres), l'augmentation du taux de carbone, du $\mathrm{C} / \mathrm{N}$ et l'apparition d'un horizon $\mathrm{Bh}$ caractéristique de la migration de la matière organique, et des coefficients de redistribution du fer et de laluminium plus élevés sous les épicéas que sous les feuillus confirment les modifications morphologiques des horizons organiques.

Il est indéniable que l'Epicéa a provoqué un début de podzolisation. 
Dans cette première partie nous avons qualifié les phénomènes et constaté que 1'Epicéa entraînait la podzolisation.

Derrière ce terme de podzolisation, il est souvent sous-entendu, pour le public, des effets de dégradation irréversible, de stérilisation du sol et d'appauvrissement. Qu'en est-il exactement?

Nous essayerons donc dans la deuxième partie de quantifier cette action de l'Epicéa par des bilans pondéraux permettant d'une part d'évaluer les réserves d'éléments minéraux échangeables et mobilisables caractérisant le niveau de fertilité de la station et sa diminution éventuelle. D'autre part de quantifier l'accélération de l'altération par l'étude des éléments totaux et des minéraux.

\section{II. - Bilans géochimiques comparés des éléments minéraux des différents compartiments (échangeables, mobilisables et totaux)}

\section{II.1. - Généralités}

Dans le cadre de cette étude deux types de bilan ont été réalisés en fonction de deux objectifs.

- Evaluer les réserves actuelles disponibles en éléments échangeables et mobilisables correspondant à l'évaluation de la fertilité de la station à court terme. C'est le bilan des éléments contenus dans la couche du sol prospectable par les racines.

- Comparer l'évolution de l'altération des minéraux du sol (par les éléments totaux) et cela par la technique du bilan isoquartz.

\section{II.11. - Principe du bilan isoquartz}

Le principe du bilan isoquartz a été défini par les géologues et adapté aux sols par Lelong, Souchier (1972), Meilhac (1970).

L'altération des composants minéraux du sol correspond à des modifications structurales des minéraux et à des pertes de matières inéluctables. Ces pertes correspondent à la variation entre la masse de la colonne du sol actuel et celle de la roche mère initiale qui lui a donné naissance.

Pour les évaluer, il suffit de disposer dans la masse du sol d'un minéral invariant, résistant à l'altération. Celui-ci sert d'étalon interne.

Dans les sols de climats tempérés le quartz est considéré comme un minéral stable, c'est pourquoi nous l'avons choisi comme étalon interne.

Nous pouvons ainsi rapporter toutes les variations des éléments chimiques étudiés au poids de quartz contenu dans la colonne de roche mère initiale et accéder à l'évolution quantitative, en vraie grandeur, de ces éléments. 


\section{II.12. - Le principe du calcul isoquartz}

Le principe est de calculer horizon par horizon, la variation en un élément par rapport à $\mathrm{C}$ horizon qui contient la même masse de quartz que lui et de sommer sur l'ensemble du profil.

La variation $(\Delta)$ pour un élément « $\mathrm{x} »$ dans un horizon « $\mathrm{i} »$ est :

$$
\Delta \mathrm{i}=\mathrm{h}_{\mathrm{i}} \mathrm{d}_{\mathrm{i}}^{\prime}\left(\mathrm{x}_{\mathrm{i}}-\mathrm{x}_{\mathrm{o}} \frac{\mathrm{q}_{\mathrm{i}}}{\mathrm{q}_{\mathrm{o}}}\right)
$$

$\begin{array}{ll}\mathrm{h}_{\mathrm{i}}=\text { épaisseur de l'horizon } \mathrm{i} & : \mathrm{q}_{\mathrm{i}}=\text { teneur en quartz dans } \mathrm{i} \\ \mathrm{d}_{\mathrm{i}}=\text { densité apparente dans } \mathrm{i} & : \mathrm{q}_{\mathrm{o}}=\text { teneur en quartz dans } \mathrm{C} \\ \mathrm{x}_{\mathrm{i}}=\text { teneur en l'élément dans } \mathrm{i} & : \mathrm{x}_{\mathrm{o}}=\text { teneur en l'élément dans } \mathrm{C}\end{array}$

$\mathrm{C}=$ horizon de référence

Remarque : dans cet article nous comparons les résultats moyens des résultats de 3 profils sous les feuillus et de 2 profils sous les épicéas.

\section{II.13. - Etablissement des bilans et leurs limites}

La première hypothèse d'homogénéité verticale du profil du sol acceptée, le bilan isoquartz a pour principe une référence à l'horizon $C$ du sol considéré pour le sol comme le matériau initial. C'est la deuxième hypothèse fondamentale du calcul.

Nous avons cependant constaté dans cette étude, et nous l'aborderons dans la suite de cet article, que cet horizon $C$ pouvait avoir lui-même subit une pédogenèse différente sous les deux peuplements donc créé une erreur supplémentaire sur l'évaluation des variations des éléments.

D'autre part l'altération réduit les réserves minérales donc la masse de matière du sol. Celle-ci est plus ou moins compensée par les apports biologiques (litières). La perte de poids crée des modifications de la densité apparente des horizons du sol.

Nous avons constaté de plus que sous les épicéas les densités apparentes des. horizons de surface étaient plus faibles que sous les feuillus. L'altération seule ne peut expliquer ces écarts. L'enracinement superficiel de l'Epicéa a pu créer ces modifications physiques de la densité : c'est ici un foisonnement.

Il est nécessaire d'évaluer ce foisonnement et de corriger l'épaisseur des profils de telle sorte que l'on compare des épaisseurs initiales du sol égales.

\section{II.14. - Evaluation du foisonnement ou du tassement}

Le bilan isoquartz permet de calculer les pertes relatives $P_{i}$ de matière minérale pour un horizon « $\mathrm{i} »$

$$
P_{i}=\left(M_{i}-M_{0} \frac{q_{i}}{q_{o}}\right) / M_{0} \frac{q_{i}}{q_{o}} \%
$$

$\mathbf{M}=$ somme des éléments minéraux « $\mathrm{x}$ » dans l'horizon 
Le taux de foisonnement $F$ est défini par :

$F=\frac{d^{\prime} c}{d^{\prime} m}$ où $d^{\prime} m$ est la densité apparente mesurée et d'c est la densité apparente calculée :

$$
d^{\prime} \mathrm{c} \text { de } \mathbf{i}=\mathrm{d}^{\prime} \mathrm{m} \text { de } \mathrm{C} \times \frac{100-\mathbf{P}_{\mathbf{i}}}{100}
$$

si $d^{\prime} c>d^{\prime} m$ : il y a foisonnement c'est-à-dire augmentation du volume du sol sans variation de masse

$\mathrm{d}^{\prime} \mathrm{c}<\mathrm{d}^{\prime} \mathrm{m}$ : il y a tassement.

Les bilans sont réalisés pour une épaisseur des profils équivalente sous les deux peuplements et correspondant à $80 \mathrm{~cm}$ de l'horizon $\mathrm{C}$ de référence soit $100 \mathrm{~cm}$ sous les épicéas et $94 \mathrm{~cm}$ sous les feuillus.

\section{II.2. - Résultat des bilans}

\section{II.21. - Stock des éléments échangeables, du carbone et de l'azote Immobilisation dans les écosystèmes}

L'accumulation de matière organique plus importante sous les épicéas a eu pour conséquence l'augmentation de la capacité totale d'échange et avec elle la potentialité de retenir plus de cations échangeables. Nous constatons en effet que les réserves en éléments échangeables sont plus abondantes sous les résineux d'environ 10 p. 100 pour le calcium et le manganèse, de $30 \mathrm{p}$. 100 pour le potassium et le magnésium.

A court terme, ces réserves d'éléments échangeables sont le refket de la fertilité du sol car ils représentent les éléments directement assimilables. Les épicéas ne provoquent donc pas au cours de la première génération de baisse de la fertilité de la station (sol sur limon acide). Ces résultats concordent avec les travaux de HolmsgaArd (1968), et GENSSLER (1959) qui constatent une aussi bonne productivité en deuxième ou troisième génération de résineux qu'en première génération.

Remarque : L'augmentation apparente de la fertilité à court terme est liée à celle de la matière organique du sol. Le peuplement d'Epicéa étudié est dense et la minéralisation de la matière organique faible. Or la seconde génération sera précédée d'une coupe à blanc étoc. Celle-ci aura pour effet de provoquer une accélération de la minéralisation du stock de matière organique et comme le montre une expérience lysimétrique dans une coupe à blanc, le lessivage des éléments minéraux sera intensifié provoquant ainsi la baisse de ces réserves en éléments assimilables.

L'établissement des biomasses puis des minéralomasses dans les deux écoystèmes permet d'évaluer les quantités d'éléments immobilisées dans les parties aériennes des végétaux et dans les litières. En quantité elles sont équivalentes aux réserves des éléments échangeables du sol. Les épicéas se différencient des feuillus par une immobilisation plus importante du carbone, de l'azote et du magnésium (tableau 2). 


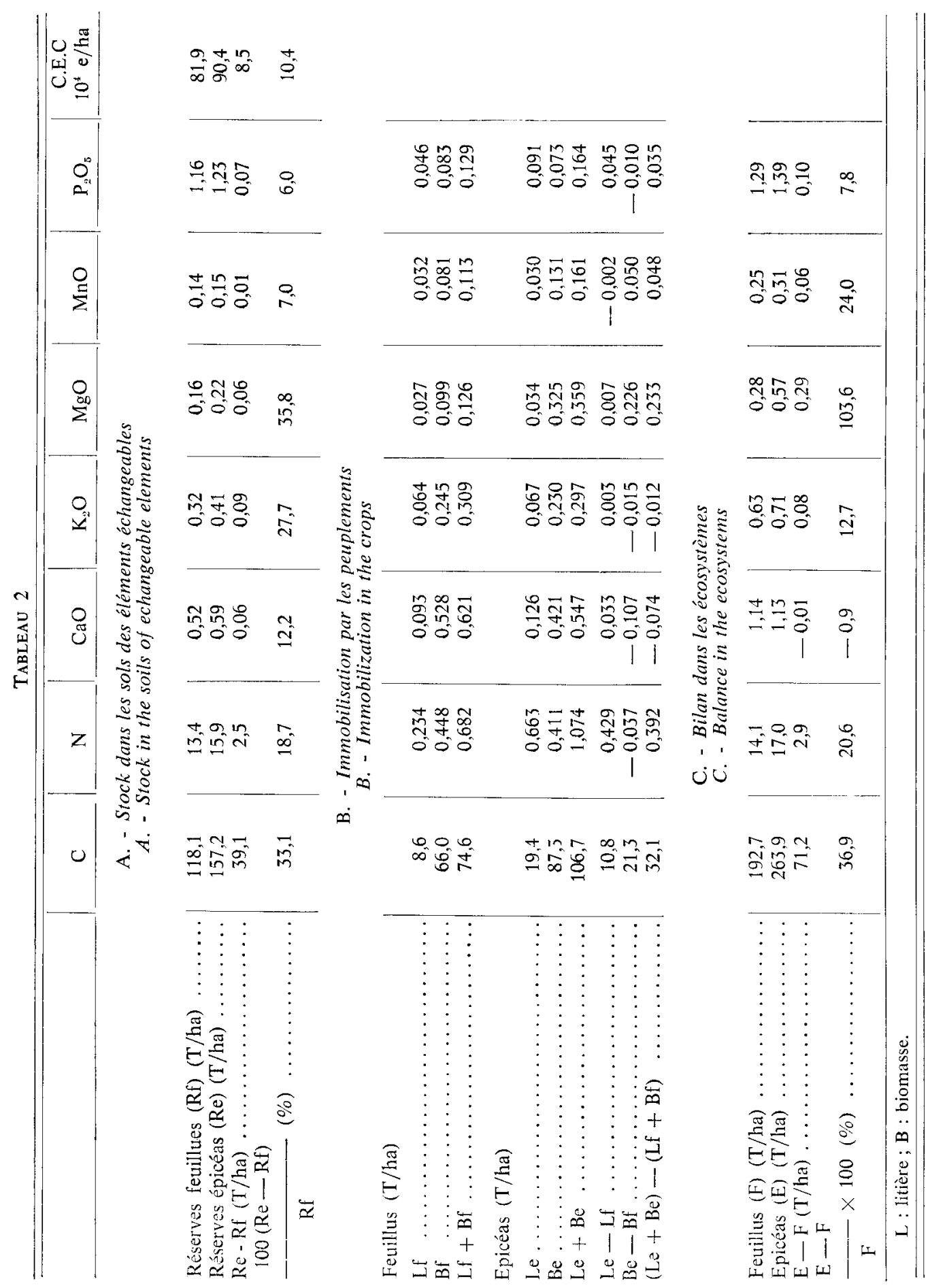


Le total des immobilisations minérales, organiques et des éléments échangeables dans l'écosystème Epicéa est supérieur à celui de l'écosystème feuillu sauf pour le calcium où il lui est égal.

\section{II.22. - Stocks des éléments mobilisables et totaux}

Les réserves en éléments totaux dans le sol caractérisent la potentialité de ce sol à long terme. Les éléments mobilisables sont susceptibles de pouvoir recharger rapidement le complexe absorbant en éléments nécessaires à une bonne nutrition des arbres. De ces réserves en éléments mobilisables dépend la fertilité à court et moyen terme de la station.

Les réserves actuelles dans les sols des deux peuplements sont du même ordre de grandeur.

\section{II.3. - Variations isoquartz}

Les variations isoquartz correspondent aux quantités de matières qui ont été entraînées hors du profil (en référence à l'horizon $C$ contenant la même quantité de quartz que lui) s'il y a perte.

S’il y a gain elles correspondent à un apport extérieur (eaux des pluies).

\section{II.31. - Variations des éléments mobilisables et du phosphore assimilable (tableau 3)}

Sous les feuillus l'altération des minéraux du sol se traduit par une augmentation du stock (complexe d'altération) des éléments mobilisables (magnésium, manganèse) et du phosphore assimilable (extraction Duchaufour-Bonneau, 1969). Le potassium mobilisable est peu retenu, sa variation est négative. Sous les épicéas les processus sont les mêmes mais leurs intensités sont plus élevées sauf pour le magnésium dont la variation est négative alors qu'elle était positive sous les feuillus.

\section{II.32. - Variations des éléments libres et amorphes}

L'horizon $\mathrm{C}$ de référence contient déjà des quantités importantes d'éléments libres ou amorphes du fait de son origine sédimentaire ou d'une altération très ancienne liée à la mise en surface. L'action des composés organiques libérés par la végétation s'est surimposée et a modifié positivement ou négativement leur quantité.

Sous feuillus l'alumine a augmenté tandis que le fer et la silice ont diminué. Sous les épicéas le fer a diminué également mais moins que sous feuillus. L'alumine a augmenté davantage que sous le taillis-sous-futaie et la variation de la silice est nettement positive alors qu'elle était négative sous feuillus.

On peut donc estimer que l'altération est plus rapide sous les épicéas. Par rapport au sol sous feuillus, les taux de variations (rapport des pertes du sol par rapport au stock initial de la roche mère dont il est issu) ont augmenté sous les résineux de 3,7 p. 100 pour le fer, de 27,4 p. 100 pour l'aluminium et de 44 p. 100 pour le silicium (tableau 3). 
TABleau 3

Bilan des éléments mobilisables, libres et amorphe's, ct du phosphore cassimilable Mobilizable, free and amorphous elements ard assimilable phosphorus

\begin{tabular}{|c|c|c|c|c|c|c|c|}
\hline \multirow[b]{3}{*}{$\begin{array}{l}\text { Réserves feuil'ues (Rf) } \\
(\mathrm{T} / \mathrm{ha}) \ldots \ldots \ldots \ldots\end{array}$} & \multirow{2}{*}{$\mathrm{P}_{2} \mathrm{O}_{5}$} & \multicolumn{3}{|c|}{ Mobilisable } & \multicolumn{3}{|c|}{ Libre + amorphe } \\
\hline & & $\mathrm{K}, \mathrm{O}$ & $\mathrm{MgO}$ & $\mathrm{MnO}$ & $\mathrm{Al}_{2 .} \mathrm{O}_{3}$ & $\mathrm{Fe}_{2} \mathrm{O}_{3}$ & $\mathrm{SiO}_{2}$ \\
\hline & 1,16 & 1,67 & 2,00 & $4,2 \mathcal{E}$ & 90,1 & 305,1 & 55,9 \\
\hline $\begin{array}{l}\text { Variation isoquartz feuillue } \\
\text { (Vf) }(\mathrm{T} / \mathrm{ha}) \ldots \ldots \ldots \ldots \ldots\end{array}$ & 0.75 & $-\quad 4,12$ & 0,10 & 1,85 & 7,6 & $-\quad 58,6$ & $-17,6$ \\
\hline $\begin{array}{l}\text { Taux de variation feuillue } \\
\text { (Tf) }(\%) \ldots \ldots \ldots \ldots \ldots\end{array}$ & 182,9 & $-71,1$ & 5,2 & 76,1 & 9,2 & $-16,1$ & $-23,9$ \\
\hline Réserves épicéas (Re) $(\mathrm{T} / \mathrm{ha})$ & 1,23 & 1,55 & 2,00 & 4,4 & 95,9 & 360,1 & 55,6 \\
\hline $\begin{array}{l}\text { Variation isoquartz épicéas } \\
\text { (Ve) }(\mathrm{T} / \mathrm{ha}) \ldots \ldots \ldots \ldots \ldots\end{array}$ & 0,82 & $=\quad 6,74$ & $-0,84$ & 1,99 & 25,7 & $-50,8$ & 14,0 \\
\hline $\begin{array}{l}\text { Taux de variation épicéas } \\
(\mathrm{Te})(\%) \ldots \ldots \ldots \ldots \ldots\end{array}$ & 200,0 & $-81,3$ & -29.6 & 82,6 & 36,6 & $-12,4$ & 33,7 \\
\hline $\mathrm{Ve}-\mathrm{Vf}(\mathrm{T} / \mathrm{ha}) \ldots \ldots \ldots$ & 0,07 & $-\quad 2.62$ & $-0,94$ & 0,14 & 18,1 & 7,8 & 31,6 \\
\hline$\frac{\mathrm{Ve}-\mathrm{Vf}}{\mathrm{Rf}} \times 100(\%)$ & 6,0 & $-156,4$ & $-46,9$ & 3.3 & 20,1 & 2,6 & 56,5 \\
\hline $\mathrm{Te}-\mathrm{Tf}(\%) \ldots \ldots$ & 17,1 & & $-34,8$ & 6,1 & 27,4 & 3,7 & 44,0 \\
\hline
\end{tabular}

Ces résultats confirment les précédents et caractérisent une dynamique podzolisante nette sous les épicéas.

Si cette dynamique podzolisante est réelle sous les épicéas cela doit se concrétiser par des pertes d’éléments totaux plus importantes sous les épicéas.

\section{II.33. - Variations des éléments totaux}

Les principaux résultats sont rassemblés dans le tableau 4.

Dans les sols des deux écosystèmes feuillus et résineux l'altération provoque une diminution des réserves des éléments totaux sauf pour deux éléments : le calcium et le manganèse. Au cours du cycle biogéochimique ces deux éléments sont rrélevés par les racines dans les couches profondes de la roche mère puis retournent au sol par les litières et sont ensuite stockés dans les horizons de surface. 


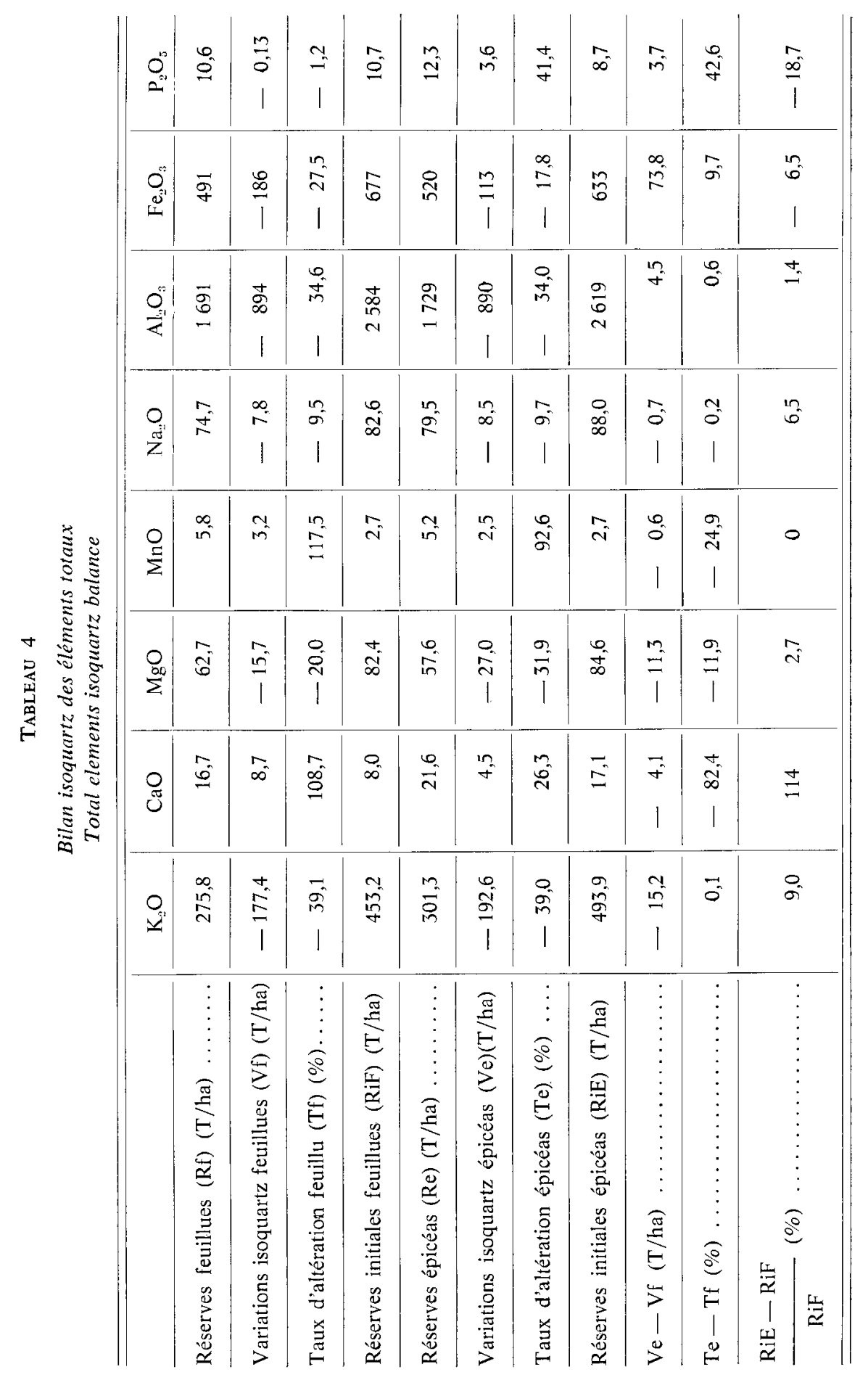


Les pertes calculées sont plus importantes sous les résineux $(\mathrm{K}, \mathrm{Mg})$ ou les gains moindres $(\mathrm{Ca}, \mathrm{Mn})$ pour tous les éléments minéraux nécessaires à l'alimentation minérale des arbres (variations isoquartz du tableau 4). Les pertes en aluminium total semblent moins importantes sous les épicéas ce qui est en contradiction apparente avec les résultats précédents. Ces résultats d'aluminium total correspondent à la somme de laluminium total de constitution des minéraux et des différentes autres formes en particulier de la forme aluminium libre (extraction Tamm + hydrosulfite de sodium). De ce fait les pertes d'aluminium total de constitution des minéraux sont plus élevées de 14,1 T/ha.

En ce qui concerne le fer total les différences importantes constatées ne peuvent s'expliquer que par une hétérogénéité. C'est ce que nous constatons d'ailleurs dans un des profils sous feuillus et en particulier pour l'horizon $\mathrm{C}$ de référence (voir Tableau 1).

Remarque: Une différence de $1 \%$ de la concentration dans l'horizon de référence $C$ provoque sous les feuillus une modification des variations de 13,7 $\mathrm{T} / \mathrm{ha}$.

\section{Conclusion}

45 ans de monoculture d'Epicéa commun sur un sol brun acide sur limon des Ardennes primaires ont provoqué des modifications des caractéristiques physicochimiques de cet ancien sol de forêt feuillue.

L'humus de type mull acide sous les feuillus s'est transformé en moder mull et un horizon $\mathrm{Bh}$ apparaît à la base du Al. Le sol brun acide est maintenant de type ocreux avec un épaississement important de la litière. Le coefficient de décomposition de la matière organique de JENNY est 2,6 fois plus élevé sous les feuillus $(24,2)$ que sous les Epicéas $(9,3)$ pour des retombées de litières quantitativement égales.

L'épicéa commun est une espèce acidifiante. Les acidités libres, d'échange et totales des horizons organiques et des eaux de gravités sont plus élevées (RicherT, 1978).

La quantité des éléments mis en circulation chez l'Epicéa est plus importante que celle du taillis-sous-futaie feuillu. Les stocks des éléments échangeables, du carbone et de l'azote sont plus élevés. Cependant la minéralisation de l'azote est moins intense que sous les feuillus (Guillaud, 1979).

Les réserves d'éléments mobilisables et d'éléments totaux sont semblables dans les deux écosystèmes. Les variations isoquartz des éléments mobilisables sont assez voisines et ne permettent pas de différencier nettement les deux stations. Par contre les pertes en éléments totaux notamment $\mathrm{K}$ et $\mathrm{Mg}$ sont, en valeur absolue, plus fortes sous l'Epicéa. Le taux de variation du potassium total sous Epicéa est peu différont de celui qui a été calculé sous feuillus, alors que pour $\mathrm{Mg}$ et $\mathrm{Mn}$ il est beaucoup plus élevé sous résineux. Ceci traduit vraisemblablement sous Epicéa une altération renforcée portant essentiellement sur les micas. Une faible perte de fertilité à long terme semble donc possible. 
Les coefficients de redistribution du fer et de l'alumine libres confirment l'action podzolisante de l'Epicéa sur ce type de substrat.

Cependant il n'est pas possible d'être très affirmatif, du fait notamment de la faible différence des taux de variation du potassium mais aussi, malgré les précautions prises dans le choix des stations, d'une certaine hétérogénéité des profils.

En effet les analyses de caractérisation de l'horizon $C$, de référence, montrent sous les épicéas une faible accumulation d'argile due peut-être à un lessivage (Tableau 1, Figure 3). Nous vérifions actuellement cette hypothèse par des observations micromorphologiques et des analyses des eaux de gravité.

L'augmentation du taux d'argile évaluée à 1 p. 100 et celle des éléments mobilisables fait que notre horizon $C$ de référence a déjà subi sous les épicéas un début d'évolution. Cet enrichissement en matière surestime les pertes calculées par la méthode du bilan isoquartz.

Nous avons jugé indispensable dans ces conditions de procéder à une nouvelle estimation des pertes d'éléments dues à une altération des minéraux en tenant compte de ce phénomène éventuel. Pour cela nous «corrigeons» l'horizon de référence sous Epicéa afin de reconstituer la composition qu'il aurait en absence de lessivage.

Les résultats corrigés, les pertes sous Epicéa restent plus importantes que sous feuillus. Elles correspondent cependant à la fois à une altération et au simple départ des argiles par lessivage. Pour obtenir les vraies pertes par altération seule il faudrait donc diminuer les chiffres du tableau 5 des quantités correspondant à l'accumulation d'argile en $\mathrm{C}$. Il est impossible de le calculer exactement car nous ne connaissons pas l'épaisseur sur laquelle a pu se faire cette accumulation.

Il est vraisemblable qu'on arriverait dans ces conditions à une très faible perte de potassium et cela correspondrait bien au fait que dans le dispositif lysimétrique qui a été installé dans ces stations la concentration en potassium des eaux n'est pas plus élevée sous Epicéa que sous feuillus.

\section{Tableau 5}

Bilan isoquartz corrigé, des éléments totaux sous les épicéas

Total elements isoquartz balance under spruce after correction

\begin{tabular}{|c|c|c|c|c|c|c|c|c|}
\hline & $\mathrm{K} \mathrm{O}$ & $\mathrm{CaO}$ & $\mathrm{MgO}$ & $\mathrm{MnO}$ & $\mathrm{Na}_{2} \mathrm{O}$ & $\mathrm{Al}_{z} \mathrm{O}_{: 3}$ & $\mathrm{Fe}_{2} \mathrm{O}_{3}$ & $\mathrm{P}_{21} \mathrm{O}_{5}$ \\
\hline $\begin{array}{l}\text { Concentration, référence } \\
\text { de l'horizon sous épicéas } \\
(\%) \quad \ldots \ldots \ldots \ldots \ldots \ldots \ldots\end{array}$ & 35,2 & 1,2 & 6,0 & 0,2 & 6,3 & 189 & 45 & 0,6 \\
\hline $\begin{array}{l}\text { Variations isoquartz } \\
(\mathrm{T} / \mathrm{ha}) \quad \ldots \ldots \ldots \ldots \ldots\end{array}$ & $-181,4$ & 4,9 & $-23,5$ & 2,5 & $-7,1$ & -845 & 一 86 & 3,4 \\
\hline $\mathrm{Ve}-\mathrm{Vf}$ & -4 & $-3,8$ & $-7,8$ & $-0,7$ & $+0,7$ & 49 & 100 & 3,5 \\
\hline
\end{tabular}

$V e=$ Variations isoquartz sous épicéas.

$\mathbf{V f}=$ Variations isoquartz sous feuillus. 
Cependant deux autres observations amènent à se demander si cette «correction de lessivage» est légitime. D'une part les eaux des lysimètres n'ont jamais montré d'argile en suspension, mais peut-être sont-elles arrêtées par les filtres des lysimètres eux-mêmes : la réponse ne pourra être donnée que lors de la destruction des lysimètres. Il se peut aussi que le lessivage ait eu lieu surtout au début de la révolution d'Epicéa alors que les études lysimétriques ne sont menées que 45 ans après la plantation.

D'autre part la correction effectuée amène un gain relativement important d'aluminium total ( 49 tonnes soit 2 p. 100 par rapport au stock actuel) alors que les calculs initiaux, sans corrections, aboutissaient sensiblement à une constance de l'aluminium ce qui était plus satisfaisant.

Malgré les précautions prises et des réflexions approfondies nous devons donc conclure que notre étude du bilan ne permet pas de conclusion formelle. Si lon ne tient pas compte du lessivage la perte de potassium total à long terme n'est pas négligeable. Dans le cas contraire elle est pratiquement nulle, mais il reste des pertes notables en magnésium et calcium. Le bilan d'alumine libre et de silice amorphe oblige à conclure à un renforcement de l'altération sous Epicéa, mais elle est loin d'être dramatique.

Cette altération conduit à des pertes qui pour le potassium semblent assez faibles et d'un ordre de grandeur identique à celui des erreurs auxquelles conduit fatalement l'inévitable manque d'homogénéité verticale des profils.

Dans l'ensemble l'influence de l'Epicéa est bien, sur les schistes des Ardennes, de même nature que celle qui a déjà été mise en évidence sur les granites du Massif Central (Brethes, Nys, 1975), où déjà l'attention avait été attirée sur l'altération des micas. Mais elle est ici, sur un matériau plus «tamponné » plus discrète et pour certains éléments, à la limite des possibilités de détection.

Comme dans le Massif Central ces phénomènes ne concernent, du point de vue de la fertilité du sol, que le très long terme. La conservation de l'essentielle des propriétés du sol étant certaine à court et moyen terme.

Reçu pour publication en janvier 1980.

\section{Summary}

\section{Changes in physico-chemical properties of a brown soil in a hardwood forest in Ardennes moutain by introduction of Norway Spruce}

The objective of our work was to determine the influence of Norway Spruce (Picea abies) plantation on soil fertility and alteration of minerals, compared with a deciduous stand (coppice with standards) in the primary part of Ardennes moutains in France. The parent rock is acid silt over schists of Revinien.

Under hardwoods the Humus is a «acid mull». It is a «moder mull» under the conifers. Acidity and amorphous elements are more important under spruce. It induces with the beginnings podzolisation.

The reserves of exchangeable and available elements in the soil and of elements in the biomass are the same in the two stands. 
We had to evaluate the quartz content of the horizons, then to calculate ponderal gains or losses of some elements and minerals in the same profile, as compared to the $\mathrm{C}$ horizon, using quartz as invariable standard.

The results show under spruce : $\mathbf{K}$ and $\mathrm{Mg}$;

- slight losses of the reserves of total elements except significant losses of total

- gains of amorphous $\mathrm{Al}, \mathrm{Fe}, \mathrm{Si}$;

- greater weathering of minerals (micas) and clay;

- slight leaching of clay ;

We debate of the validity of isoquartz balance.

The stability of the reserves of exchangeable and available element, may by explained by the supply to the soil exchange-complex of elements removed from silicates by faster weathering.

\section{Références bibliographiques}

Barshad I., Chemistry of the soil. II Editions Firman E. Bear.

Bonneau M., 1973. Les enrésinements risquent-ils de diminuer la fertilité des sols. Rev. for. fr., XXV, 4.

Bonneau M., Brethes A., Nys C., Souchier B., 1976. Influence d'une plantation d'Epicéa sur un sol du Massif Central. Lejeunia $\mathrm{n}^{\circ}$ 82, nov.

Bonneau M., 1978. Conséquences pédologiques des enrésinements en forêt. Académie d'agriculture de France, p. 931.

Bonneau M. et al., 1979. Effets de boisements résineux purs sur l'évolution de la fertilité du sol. Rev. for. fr., XXXI, 3, 198-207.

Brethes A., Nys C., 1975. Effets des résineux sur la fertilité des sols. Difficultés des recherches et premiers résultats. Bull. A.F.E.S. $\mathrm{n}^{\circ} 1$.

Duchaufour Ph., 1970. Précis de Pédologie. Masson, Paris, 481 p.

Duchaufour Ph., Bonneau M., 1961. Evolution d'un sol de forêt feuillue provoquée par une plantation de Douglas d'une treniaine d'années. Rev. for. fr., p. 793.

Duchaufour Ph., Bonneau M., 1969. Une méthode nouvelle de dosage du phosphore assimilable dans les sols forestiers. Bull. A.F.E.S. $\mathrm{n}^{\circ} 4$.

GeNSSLER H., 1959. Veränderungen von Boden und Vegetation nach generationsweisen Fichtenanbau. Thèse Hann-Münden.

Guillaud J., 1979. Etude comparée du cycle biogéochimique de l'azote dans deux écosystèmes forestiers feuillu et résineux des Ardennes primaires. Mémoire E.N.I.T.E.F., Publication C.N.R.F.

Holmsgaard F., 1968. Ertragskundliche Untersuchungen in Fichtenbeständen erster und zweiter Generation im dänischen Jungmoränengebiet. Tagungsberichte der Deutsche Akademie der Landswirtschaft vissenschaften in Berlin, $\mathrm{n}^{\circ} 84$.

JACKSON M.L., 1974. Soil chemical analysis advanced course. Prentice Hall (U.S.A.), 895 p.

Lelong F., Souchier B., 1972. Comparaison de bilans d'altération sur roches granitiques en zone tempérée et en zone équatoriale. C.R. Acad. Sci. Paris, Série D, 274, 1896.

Manil G., 1966. Le problème de la conservation de la fertilité des sols sous monoculture de résineux. Comptes rendus du $6^{\circ}$ congrès forestier mondial. Vol. II, Madrid, p. 2239.

Meilhac A., 1970. Origine et évolution des minéraux phylliteux dans les altérations de granites en pays tempéré. Bilan minéralogique et géochimique sur deux massifs des Vosges. Thèse $3^{\mathrm{e}}$ cycle, Strasbourg, $71 \mathrm{p}$.

Miehlich G., 1970. Veränderung eines Lösslehm. Pseudogley durch Fichtenreianbau. Thèse, Hambourg. 
NiHLgard B., 1972. Plant biomass, primary production and distribution of chemical elements in a beech and a planted spruce forest in South Sweden. O.I.K.O.S., 23 (I), 69.

Noirfalise A., Vanessa R., 1975. Conséquences de la monoculture des conifères pour la conservation des sol: et le bilan hydrologique. Centres d'écologie forestière, Association des espaces verts, Bruzelles.

Nys C., 1976. Fiude comparative de l'évolution des sols sous des peuplements feuillus (Hêtre) ¿i résineux (Douglas, Epicéa) dans le Morvan. Publication C.N.R.F., Nancy, $20 \mathrm{p}$.

Paternoster M., 1979. Etude comparè sios éléments en solution dans les eaux de gravité de deux écosystèmes forestiers feuillu et résineux des Ardennes primaires. D.E.A. Université Nancy, 55 p.

Richert D., 1978. Etude de l'influence des résineux sur l'acidité des sols et des eaux. Mémoire E.N.I.T.E.F. Publication C.N.R.F., 73 p.

ScILLENKER et al., 1969. Untersuchungen über Auswirkungen des Fichtenreinanbaus auf Parabraunerden und Pseudogleys des Neckarlandes. Mitteilungen dos Vereins für fortliche Standortkunde, n"19, p. 72.

Souchier B., 1971. Evolution des sols sur roches cristallines à l'étage montagnard (Vosges). Thèse d'Etat, Université de Nancy, $130 \mathrm{p}$.

VEDY J.C., 1973. Relation entre le cycle biogéochimique des cations et l'humidification en milieu acide. Thèse doctorat d'Etat, Nancy I, $116 \mathrm{p}$.

Compte rendu D.G.R.S.T., 1977. Modification de fertilité des sols sous boisements artificiels de résineux purs. Publication C.N.R.F., 90 p.

Ranger J., Nys C., Ranger D. Biomasse aérienne d'un taillis-sous-futaie des Ardennes françaises. Ann. Sci. for. (sous presse).

Nys C., Paternoster M., Vedy J.C. Transfert et redistribution des éléments en solution par les eaux de gravité de deux écosystèmes feuillus et résineux sur schistes du Revinien. Colloque C.N.R.S. Nancy, sept. 1979 (sous presse). 